\title{
Experimental investigation of saturation effect on pump-to-signal intensity modulation transfer in single-pump phase-insensitive fiber optic parametric amplifiers
}

Cristofori, Valentina; Lali-Dastjerdi, Zohreh; Lund-Hansen, Toke; Peucheret, Christophe; Rottwitt, Karsten

Published in:

Optical Society of America. Journal B: Optical Physics

Link to article, DOI:

10.1364/JOSAB.30.000884

Publication date:

2013

Document Version

Publisher's PDF, also known as Version of record

Link back to DTU Orbit

Citation (APA):

Cristofori, V., Lali-Dastjerdi, Z., Lund-Hansen, T., Peucheret, C., \& Rottwitt, K. (2013). Experimental investigation of saturation effect on pump-to-signal intensity modulation transfer in single-pump phase-insensitive fiber optic parametric amplifiers. Optical Society of America. Journal B: Optical Physics, 30(4), 884-888.

https://doi.org/10.1364/JOSAB.30.000884

\section{General rights}

Copyright and moral rights for the publications made accessible in the public portal are retained by the authors and/or other copyright owners and it is a condition of accessing publications that users recognise and abide by the legal requirements associated with these rights.

- Users may download and print one copy of any publication from the public portal for the purpose of private study or research.

- You may not further distribute the material or use it for any profit-making activity or commercial gain

- You may freely distribute the URL identifying the publication in the public portal 


\title{
Experimental investigation of saturation effect on pump-to-signal intensity modulation transfer in single-pump phase-insensitive fiber optic parametric amplifiers
}

\author{
Valentina Cristofori, ${ }^{1, *}$ Zohreh Lali-Dastjerdi, ${ }^{1}$ Toke Lund-Hansen, ${ }^{1,2}$ Christophe Peucheret, ${ }^{1}$ \\ and Karsten Rottwitt ${ }^{1}$ \\ ${ }^{1}$ DTU Fotonik, Department of Photonics Engineering, Technical University of Denmark, Lyngby DK-2800 Kgs., Denmark \\ ${ }^{2}$ Current address: FOSS Analytical A/S, Slangerupgade 69, Hillerød DK-3400, Denmark \\ ${ }^{*}$ Corresponding author: vari@fotonik.dtu.dk
}

Received November 20, 2012; revised February 13, 2013; accepted February 13, 2013; posted February 14, 2013 (Doc. ID 179961); published March 12, 2013

\begin{abstract}
We present an experimental characterization of how signal gain saturation affects the transfer of intensity modulation from the pump to the signal in single-pump, phase-insensitive fiber optic parametric amplifiers (FOPAs). In this work, we demonstrate experimentally for the first time, to our knowledge, how gain saturation of a FOPA reduces the noise contribution due to the transfer of pump power fluctuations to the signal. In a particular example, it is shown that the transferred noise is significantly reduced by a factor of 3 , while the FOPA gain remains above $10 \mathrm{~dB}$. (c) 2013 Optical Society of America
\end{abstract}

OCIS codes: $\quad 190.4970,060.2320$.

\section{INTRODUCTION}

Fiber optic parametric amplifiers (FOPAs) are attractive because they can provide large gain at arbitrary wavelengths, depending only on the availability of a suitable pump laser and fulfillment of proper phase matching conditions [1]. In addition, FOPAs are suitable for realization of all-optical signal processing functionalities, including wavelength conversion, optical switching, regeneration, phase conjugation, etc. [2]. Moreover, FOPAs can operate with a potentially low noise figure (NF) above the quantum-limited value of $3 \mathrm{~dB}$ for high-gain amplifiers when operating as phase-insensitive amplifiers. In particular, a NF below $4 \mathrm{~dB}$ has been experimentally demonstrated [3] for a phase-insensitive configuration. For phasesensitive configurations, the $\mathrm{NF}$ can be lower than $3 \mathrm{~dB}$ and a record value of $1 \mathrm{~dB}$ has been reached experimentally [4]. To date, four different noise sources have been reported, namely amplified quantum noise, residual pump noise, Ramanseeded spontaneous emission, and pump-transferred noise [5] When applying a good pump filtering, the last two contributions have been identified as the major noise factors limiting the performance of FOPAs. Moreover, typically the main region of interest in single-pump FOPAs is at the maximum gain wavelengths where the power flow from the pump to the signal is maximum and the gain depends exponentially on the product of the pump power, the fiber nonlinear coefficient, and the fiber length. For typical single-pump FOPAs with broad bandwidths on the order of tens of nanometers, the Raman-seeded spontaneous emission may be significant at the edges of the gain spectrum, while it is generally insignificant around the maximum gain wavelength and most often can be neglected in these regions. The pump transferred noise, i.e., the intensity fluctuations of the pump transferred to the signal, is significant at the maximum gain wavelengths and consequently becomes the major noise contribution. In fact, the amount of transferred noise, in case of undepleted pumps, depends on the derivative of the signal gain with respect to pump power [ $[6]$, resulting in an exponential dependence of the intensity modulation transfer (IMT) of the pump to the signal on the pump power. Furthermore, due to the ultrafast response (few fs) of the parametric process, the intensity fluctuations of the pump instantaneously modulate the gain and are thus transferred to the amplified signal, degrading the performance of the parametric amplifier. Previously, the low frequency pump-to-signal IMT has been investigated in unsaturated FOPAs, where it was shown that the intensity modulation of the signal could reach 10 times that of the pump [6]. On the other hand, it has been experimentally demonstrated that saturated FOPAs may work as power level equalizers [7,8], reducing both the impact of the spontaneous emission added to the signal by an erbium-doped fiber amplifier (EDFA) at the input of the FOPA, and the beat noise, i.e., the intrinsic noise coming from the interference between the signal and the spontaneous emission inside the FOPA. This reduction is understood by considering that, in the saturation regime, the higher signal power levels are clamped at the FOPA output, while the lower signal levels are amplified, producing a compression of the power fluctuations in the amplified signal waveform.

Considering the impact on the total noise due to the pumptransferred noise in FOPAs and the saturation effect making the FOPAs work as power level equalizers, an experimental characterization of the IMT from the pump to the signal and an investigation of its dependence on the input signal power are essential. It is well known that, in the linear regime, 
the overall noise figure of FOPAs increases with increasing signal input power [9]. However, the dependence of the IMT contribution on the signal input power has remained largely unexplored so far. In this work, the low-frequency (up to $10 \mathrm{GHz}$ ) IMT in saturated single-pump FOPAs is experimentally investigated for the first time over the entire signal gain bandwidth, showing the impact of the wavelength dependence of both IMT and gain saturation [8]. In the particular FOPA configuration which is investigated experimentally, a reduction by a factor of 3 of the IMT is achieved by increasing the signal power, resulting in the intensity fluctuations of the amplified signal being less than twice those of the pump. In addition, measurements of the noise spectral density are also presented and discussed, due to the already mentioned strong dependence of the noise performance on the input signal power in FOPAs. The noise spectral density includes all the four main contributions: the amplified spontaneous emission, including the pump residual noise and the amplified quantum noise [5]; the Raman-seeded spontaneous emission [5]; the pump fluctuations transferred to the signal [6]; and the amplified spontaneous emission introduced by the EDFA amplifying the signal at the input of the FOPA.

The article is organized as follows. In Section 2 , the experimental characterization of the IMT is introduced and the setup is described. The experimental results on the signal input power and signal wavelength dependence of the IMT are presented in Section 3, where the relative behavior of the IMT and the amplifier noise spectral density is also discussed. Finally, a conclusion is provided in Section 4 .

\section{EXPERIMENT}

The pump-to-signal IMT is characterized by applying a small amount of modulation to the pump power, corresponding to about $1 \%$ of the average power, and subsequently evaluating the temporal variations of the output signal. The IMT coefficient $\rho$ is introduced to quantify the IMT [6]. $\rho$ is defined as the ratio between the modulation index of the amplified signal $m_{s}$ and the modulation index of the input pump $m_{p}$,

$$
\rho=\frac{m_{s}}{m_{p}}
$$

where the modulation index of the pump and the signal, respectively, is calculated as

$$
m_{i}=\frac{P_{i}^{\max }-P_{i}^{\min }}{P_{i}^{\text {avg }}}=\frac{\Delta P_{i}}{P_{i}^{\text {avg }}} .
$$

In Eq. (2), $P_{i}$ is the output signal power $(i=s)$ or the input pump power $(i=p)$, and $P_{i}^{\text {avg }}$ is the corresponding average value of the power.

The experimental setup is shown in Fig. 1. A continuous wave (CW) tunable laser source (TLS) is used as pump source $\left(\lambda_{p}=1564 \mathrm{~nm}\right)$ and is subsequently phase modulated using three combined radio-frequencies (RFs) to broaden its spectrum in order to reduce the stimulated Brillouin scattering (SBS) of the pump. The pump is then intensity modulated with a $10 \mathrm{GHz}$ sinusoidal radio-frequency signal in a Mach-Zehnder modulator with a $28 \mathrm{GHz}$ bandwidth to impose intensity fluctuations. The modulation strength is chosen such that $m_{p}=0.01$. The frequencies of the RF signals used for SBS suppression $\left(f_{1}=100 \mathrm{MHz}, f_{2}=300 \mathrm{MHz}\right.$ and $f_{3}=600 \mathrm{MHz}$ ) are distinct from the amplitude modulation frequency imposed on the pump in order not to impact the results. After being intensity modulated, the pump is amplified to $0.56 \mathrm{~W}$ in an EDFA and a tunable optical bandpass filter (TBPF1) with a $2 \mathrm{~nm}$ full width at half-maximum bandwidth is used to suppress the out-of-band amplified spontaneous emission introduced by the EDFA. Another CW TLS is used as signal source and the signal power is amplified by an EDFA to be able to saturate the FOPA using different input power levels, up to $9 \mathrm{dBm}$. The maximum input power enables more than $3 \mathrm{~dB}$ signal gain reduction when compared against the unsaturated gain.

The pump and signal are then combined via a $10 \mathrm{~dB}$ coupler in a $400 \mathrm{~m}$ long highly nonlinear fiber (HNLF), with a zero-dispersion wavelength of $1559.2 \mathrm{~nm}$, a nonlinear coefficient of $11.9 \mathrm{~W}^{-1} \cdot \mathrm{km}^{-1}$, and a dispersion slope of $0.018 \mathrm{ps} /\left(\mathrm{nm}^{2} \cdot \mathrm{km}\right)$. To determine the IMT coefficient, the pump modulation index is measured at the output of the intensity modulator and subsequently compared to the modulation index of the amplified signal measured at the output of the HNLF. The output signal is selected by means of a fiber Bragg grating (FBG) used in transmission as a notch filter centered at the pump wavelength, followed by an optical bandpass filter (TBPF2) tuned to the signal wavelength. The modulation indices are measured with a network analyzer (NA) after detection by a photodiode (PD) with a $45 \mathrm{GHz}$ bandwidth. The peak-to-peak output power variation $\Delta P_{i}$ is extracted from the S-parameters provided by the NA, while the average power $P_{i}^{\text {avg }}$ is obtained from the average value of the photocurrent generated by the high-speed PD.

The measurements related to the noise spectral density $P_{\text {noise }}$ in units of $[\mathrm{dBm} / \mathrm{hm}]$ at the FOPA output are obtained by means of an optical spectrum analyzer (OSA) connected directly to the output of the HNLF. The noise spectral density was measured with an interpolation technique at the $\pm 1 \mathrm{~nm}$ offset interpolation wavelengths.

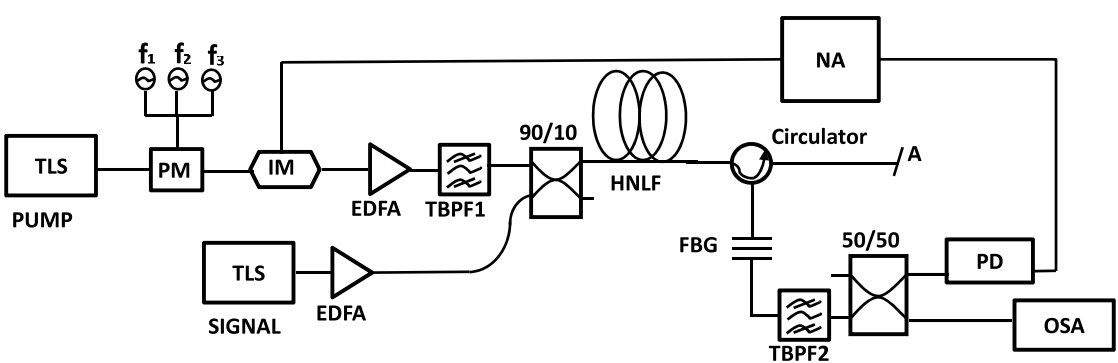

Fig. 1. Experimental setup of the FOPA for pump-to-signal IMT characterization in pump depletion. 


\section{RESULTS}

At the maximum gain wavelength $\left(\lambda_{s}=1550 \mathrm{~nm}\right)$, the output power is measured for both pump and signal as a function of the signal input power. The results are shown in Fig. 2 . As expected [8], when the signal input power is increased, the pump output power starts to decrease, and the signal output power saturates.

Due to the wavelength dependence of both IMT and gain saturation $[\underline{6}, \underline{8}]$, a characterization over the entire gain bandwidth is necessary. In particular, the gain as a function of the signal wavelength for different signal input power levels is shown in Fig. 3(a), along with results of numerical simulations. All the simulated data are obtained using a split-step Fourier method. The figure shows a decrease of the amplifier gain as the signal power is increased from $-10 \mathrm{dBm}$

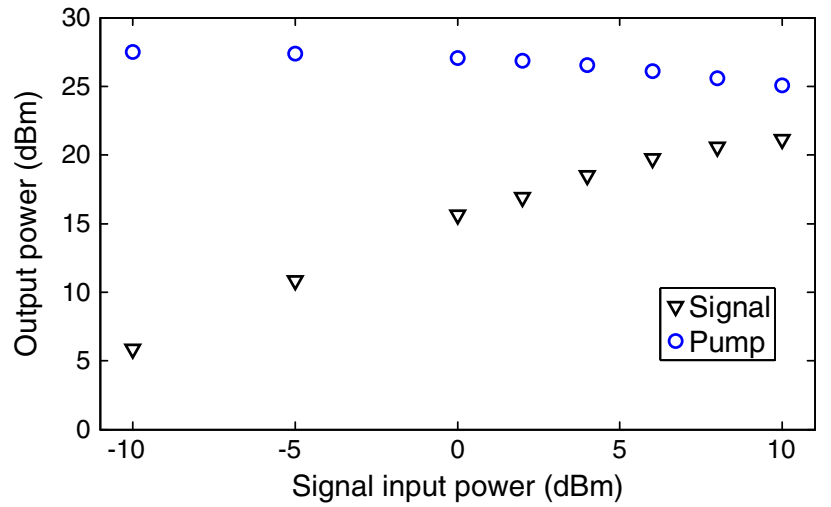

Fig. 2. (Color online) Pump and signal output power as a function of signal input power measured at the maximum gain wavelength $\left(\lambda_{s}=1550 \mathrm{~nm}\right)$.

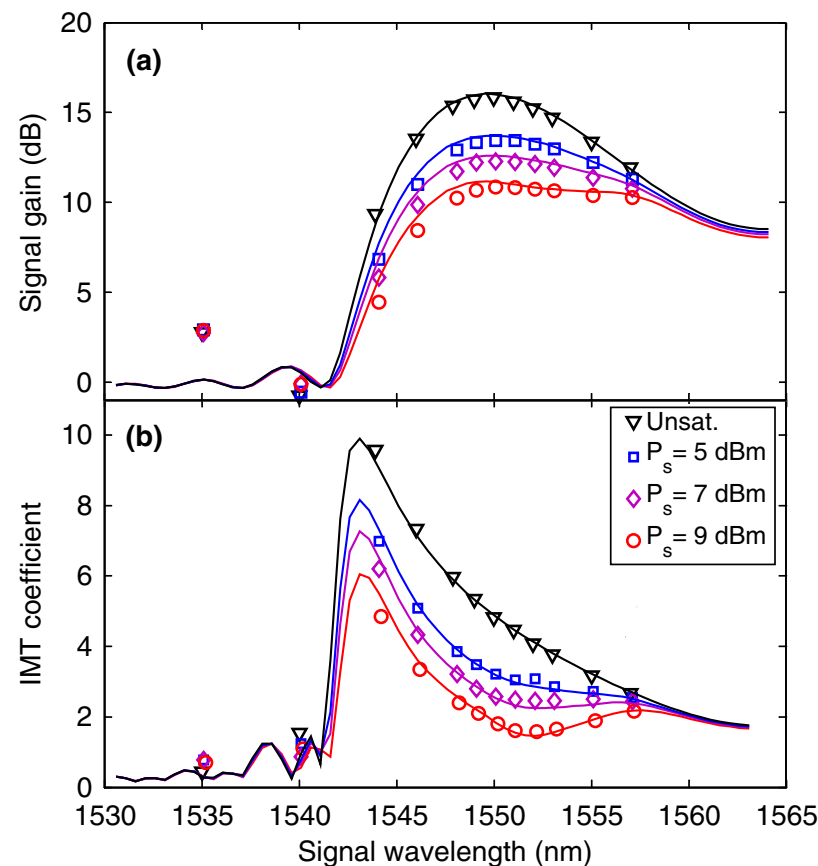

Fig. 3. (Color online) (a) Wavelength dependence of the signal gain for different signal power levels. (b) IMT coefficient $\rho$ as a function of signal wavelength for different signal power levels for $10 \mathrm{GHz} R F$ modulation frequency. The marks are the measured data and the lines are the numerical simulation results. (unsaturated regime) up to $9 \mathrm{dBm}$, confirming the gain saturation predicted from the decreasing output power in Fig. 2. The saturation effect is notable already for $5 \mathrm{dBm}$ signal power and it becomes stronger when the input signal power increases up to $9 \mathrm{dBm}$, at which point the maximum gain has decreased from the unsaturated value of 15.8 to $11 \mathrm{~dB}$. The flattening effect on the gain spectrum due to saturation is also noticed in the figure.

The corresponding IMT coefficients are shown in Fig. 3(b). As expected from the theory [6], the IMT coefficient increases with increasing wavelength separation between the signal and the pump, and the transfer is significant (i.e., the modulation index of the signal is almost 5 times the modulation index of the pump in the undepleted regime) at the peak gain wavelength. One important observation from the experimental data is that the IMT coefficient decreases when the signal input power increases and the gain saturates. Consequently the amount of fluctuations transferred to the amplified signal due to pump power fluctuations is significantly reduced. A good agreement is found between the measurements and the simulations. The good agreement validates our numerical model and hence allows us to predict gain and IMT at other pump power levels, as it is shown in the following subsection.

The usual operation region of single-pump FOPAs is around the wavelengths of maximum gain, where the maximum efficiencies in terms of energy conversion from the pump to the signal and idler are obtained. Therefore, the gain and IMT as a function of signal input power are studied in more details at the gain peak (1550 nm) and the results are shown in Figs. 4(a) and $4(\mathrm{~b})$, respectively. The IMT coefficient around the maximum gain wavelength is reduced almost a factor of 3 , from 4.85 to 1.80 , when the signal power is increased from the unsaturated regime to $P_{s}=9 \mathrm{dBm}$, i.e., the FOPA is $5 \mathrm{~dB}$

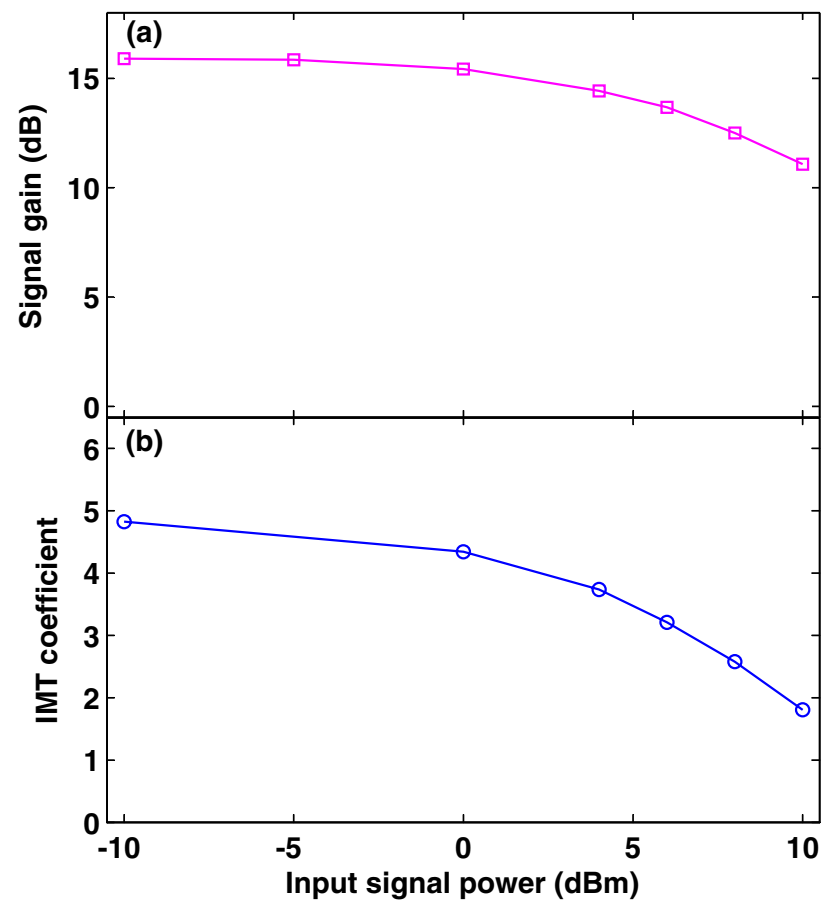

Fig. 4. (Color online) (a) Measured signal gain and (b) measured IMT coefficient as a function of the input signal power at the maximum gain $\left(\lambda_{s}=1550 \mathrm{~nm}\right)$. The $\mathrm{RF}$ modulation frequency is $10 \mathrm{GHz}$. The continuous line is a fit to the measured data. 
in depletion. Moreover, it is important to notice that the IMT is reduced below a factor of 2 at $(1550 \mathrm{~nm})$, while the amplifier gain still remains above $10 \mathrm{~dB}$. Consequently, it is possible to obtain an amplifier with a significantly lower noise contribution due to the IMT between the pump and the signal when the FOPA is operating in depletion as compared to undepletion. However, there are two main issues when operating in the saturation regime: (i) reduced gain and (ii) increased noise spectral density.

(i) The pump depletion results in a gain reduction, especially at the gain peak, creating the necessity to find a compromise between the reduction of the gain and that of the IMT. However, it should also be taken into account that the gain in parametric amplifiers depends on many factors through which it is possible to optimize the signal gain, for example the pump power or the fiber parameters. Thus it is possible to control the trade-off between IMT and signal gain reduction. In fact, considering that higher pump power provides higher gain, hence stronger saturation with increasing signal input power, it is expected that the saturation effect on the IMT will be stronger when increasing the pump power. To investigate this, we consider a pump power level for which the $3 \mathrm{~dB}$ reduction of the saturated gain is equal to $16 \mathrm{~dB}$, i.e., the nonsaturated gain of the amplifier with pump power of $27.5 \mathrm{dBm}$ studied experimentally. This can be obtained for a pump power of $P_{p}=28.1 \mathrm{dBm}$ and a signal input power of $4 \mathrm{dBm}$, as seen in the simulated gain curves of Fig. $\underline{5}$. To investigate the trade-off between gain and IMT reduction, a set of numerical calculations of the gain and the IMT as a function of the wavelength was performed for these two values of pump power, fixing the signal input power at two different levels as well, $P_{s}=-30 \mathrm{dBm}$ and $P_{s}=4 \mathrm{dBm}$, in order for the amplifier to operate in the unsaturated and saturated regime, respectively. The results for the gain and the IMT coefficient are shown in Figs. 6(a) and 6(b), respectively. As expected, the increase of the pump power level produces a higher reduction of both the gain and the IMT coefficient, due to higher pump depletion. In particular, Fig. 6(b) shows that for a pump power level of $28.1 \mathrm{dBm}$, the IMT coefficient is decreased down to 3 , which is a value lower than the one calculated for a pump power of $27.5 \mathrm{dBm}$. The improvement is also seen considering the measured data in Fig. 3(b). In fact, it is noticed that the IMT coefficient for a pump power of

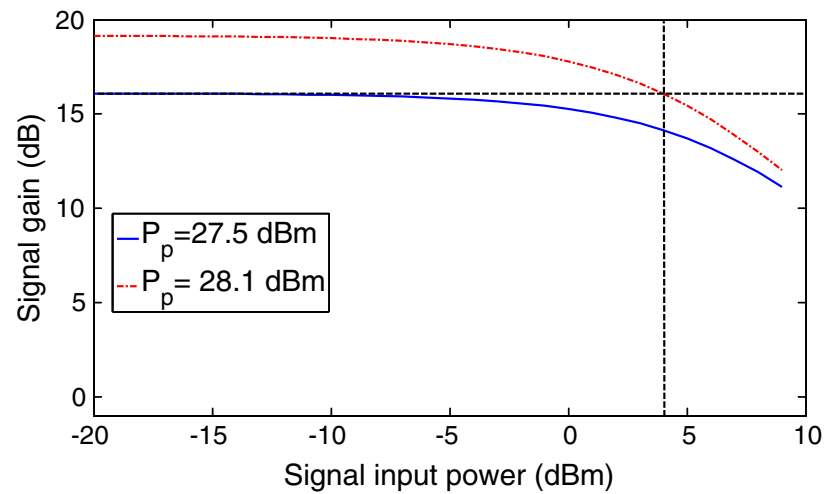

Fig. 5. (Color online) Calculated signal gain as a function of the input signal power at $\lambda_{s}=1550 \mathrm{~nm}$ for two different pump power levels $\left(P_{p}=27.5 \mathrm{dBm}, 28.1 \mathrm{dBm}\right)$.

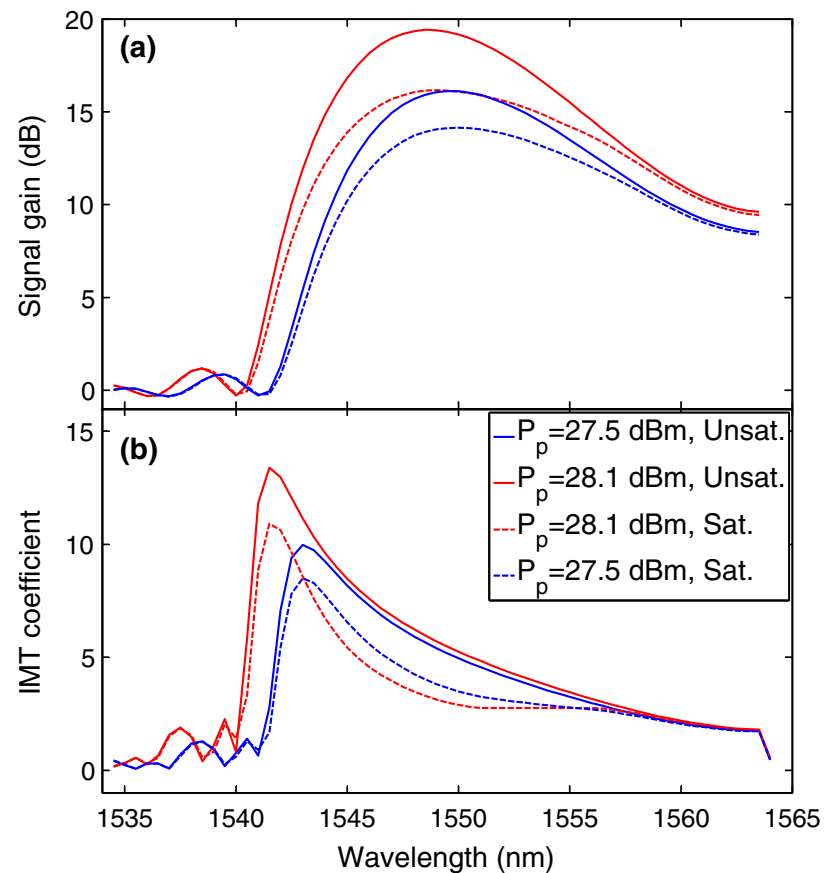

Fig. 6. (Color online) (a) Calculated signal gain and (b) calculated IMT coefficient as a function of wavelength for two different pump power levels $\left(P_{p}=27.5 \mathrm{dBm}, 28.1 \mathrm{dBm}\right)$, in both cases of unsaturated and saturated amplifier operation. The RF modulation frequency is $10 \mathrm{GHz}$.

$27.5 \mathrm{dBm}$ and a signal power of $5 \mathrm{dBm}$ is higher than 3 , showing that it is possible to obtain a lower IMT coefficient with a lower signal input power by adjusting the pump power by about $0.6 \mathrm{~dB}$.

(ii) The second issue is that the noise performance of the amplifier, i.e., amplified quantum noise, residual pump noise, Raman-seeded spontaneous emission, transferred IMT, and spontaneous emission added to the signal by the EDFA before the FOPA, worsen when the signal input power increases [9]. To characterize this, the noise spectral density $P_{\text {noise }}$ has been measured both as a function of the signal wavelength over the entire signal gain bandwidth and as a function of the signal input power at the wavelength of the gain peak. The measurements are performed for an optical signal to noise ratio (OSNR) of the pump OSNR Pump $\approx 50 \mathrm{~dB}$ and an OSNR $_{\text {Signal }}$ at the input ranging from $56.2 \mathrm{~dB}$ at maximum signal input power to $55.5 \mathrm{~dB}$ at minimum signal input power. The results are shown in Figs. 7(a) and 7(b), respectively. The measurements confirm that the noise spectral density in the amplifier increases strongly with the signal input power, reaching values of up to $-14 \mathrm{dBm} / \mathrm{nm}$ for a high pump depletion at a signal input power level of $9 \mathrm{dBm}$. A saturation of the noise spectral density is seen in Fig. 7(b) for increasing signal input power, which is due to the saturating gain. In summary, it has been shown that the IMT decreases in saturated FOPAs, making operation in saturation attractive for applications for which the intensity fluctuations of the signal constitutes a limitation of the performances [10]. However, the operation in pump depletion results in increased noise spectral density and such increases may limit the potential benefit coming from the saturation effect. As a consequence, even if the saturationinduced gain reduction may not be a major limitation for some optical signal processing applications for which a high 

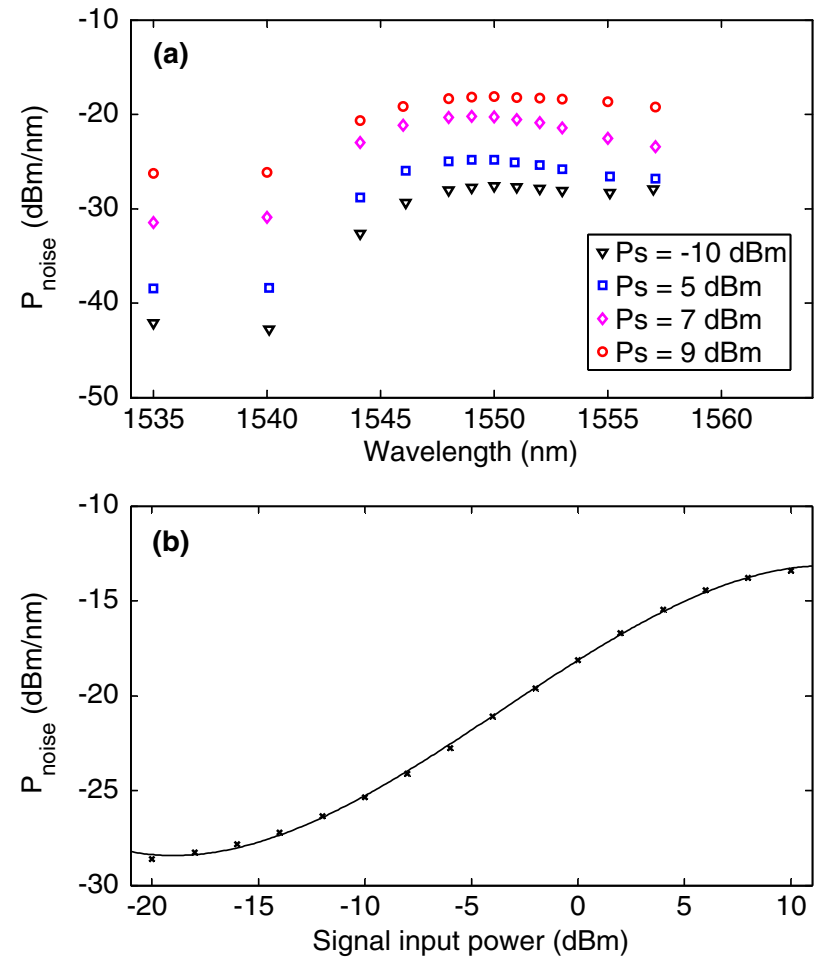

Fig. 7. (Color online) (a) Measured noise spectral density as a function of the signal wavelength for different signal power levels and (b) the noise spectral density as a function of the input signal power at the maximum gain wavelength $\left(\lambda_{s}=1550 \mathrm{~nm}\right)$.

gain is not necessary, the increase in the amplifier spectral noise density may be a fundamental constraint.

\section{CONCLUSION}

In this paper, the IMT from the pump to the signal in saturated FOPAs has been experimentally investigated and a good agreement between simulations and measurements has been demonstrated. It has been shown that the saturation effect produces a reduction of the IMT coefficient. In the particular FOPA considered in this work, the IMT is reduced below 2, thereby resulting in amplifiers with a significantly lower noise contribution from pump power fluctuations transferred to the signal. The gain is also reduced as a consequence of pump depletion. Nevertheless, it has been shown that a compromise between gain and IMT reduction could be achieved by adjusting, for example, the pump power to a higher level to obtain the same gain with a lower IMT coefficient. However, an investigation on the noise spectral density has shown that, in saturation, the other noise contributions of the amplifier, i.e., amplified spontaneous emission, Raman seeded spontaneous emission, and amplified spontaneous emission of the signal EDFA, cannot be neglected. In fact, even though the IMT decreases with increasing signal input power, the noise spectral density of the amplifier increases, which may limit the benefit due to the IMT reduction on the performance of FOPAs.

\section{ACKNOWLEDGMENTS}

OFS Fitel Denmark is acknowledged for providing the HNLFs. The Danish Research Council for Technology and Production Sciences is acknowledged for financial support (project 09-066562).

\section{REFERENCES}

1. G. P. Agrawal, Nonlinear Fiber Optics (Academic, 2006).

2. J. Hansryd, P. A. Andrekson, M. Westlund, J. Li, and P. Hedekvist, "Fiber-based optical parametric amplifiers and their applications," IEEE J. Sel. Top. Quantum Electron. 8, 506-520 (2002).

3. P. L. Voss and P. Kumar, "Raman-induced noise figure limit for $\chi^{3}$ parametric amplifiers," Opt. Lett. 29, 445-447 (2004).

4. Z. Tong, C. Lundström, P. A. Andrekson, C. J. McKinstrie, M. Karlsson, D. J. Blessing, E. Tipsuwannakul, B. J. Puttnam, H. Toda, and L. Grüner-Nielsen, "Towards ultrasensitive optical links enabled by low-noise phase-sensitive amplifiers," Nat. Photonics 5, 430-436 (2011).

5. Z. Tong, A. Bogris, M. Karlsson, and P. A. Andrekson, "Full characterization of the signal and idler noise figure spectra in single-pumped fiber optical parametric amplifiers," Opt. Express 18, 2884-2893 (2010).

6. M. E. Marhic, G. Kalogerakis, K. K. Wong, and L. G. Kazovsky, "Pump-to-signal transfer of low-frequency intensity modulation in fiber optic parametric amplifiers," J. Lightwave Technol. 23, 1049-1055 (2005).

7. K. Inoue, "Optical level equalisation based on gain saturation in fibre optical parametric amplifier," Electron. Lett. 36, 1016-1018 (2000).

8. K. Inoue and T. Mukai, "Signal wavelength dependence of gain saturation in a fiber optical parametric amplifier," Opt. Lett. 26, 10-12 (2001).

9. P. Kylemark, M. Karlsson, and P. A. Andrekson, "Gain and wavelength dependence of the noise figure in fiber optical parametric amplification," IEEE Photon. Technol. Lett. 18, 1255-1257 (2006).

10. C. Peucheret, M. Lorenzen, J. Seoane, D. Noordegraaf, C. V. Nielsen, L. Grüner-Nielsen, and K. Rottwitt, "Amplitude regeneration of RZ-DPSK signals in single-pump fiber-optic parametric amplifiers," IEEE Photon. Technol. Lett. 21, 872-874 (2009). 